\title{
SOME EXTENSIONS OF THE FOUR VALUES THEOREM OF NEVANLINNA-GUNDERSEN
}

\author{
DuC QuANG Si
}

\begin{abstract}
Nevanlinna showed that two distinct non-constant meromorphic functions on $\mathbf{C}$ must be linked by a Möbius transformation if they have the same inverse images counted with multiplicities for four distinct values. Later on, Gundersen generalized the result of Nevanlinna to the case where two meromorphic functions share two values ignoring multiplicity and share other two values with counting multiplicities. In this paper, we will extend the results of Nevanlinna-Gundersen to the case of two holomorphic mappings into $\mathbf{P}^{n}(\mathbf{C})$ sharing $(n+1)$ hyperplanes ignoring multiplicity and other $(n+1)$ hyperplanes with multiplicities counted to level 2 or $(n+1)$.
\end{abstract}

\section{Introduction}

For a divisor $v$ on $\mathbf{C}$, we define the truncated counting function of $v$ as follows:

$$
n(t, v)=\sum_{|z|<t} v(z), \quad(t>1) \quad \text { and } \quad N(r, v)=\int_{1}^{r} \frac{n(t, v)}{t} d t, \quad(r>1) .
$$

For a positive integer $k$ (maybe $k=+\infty$ ), we denote by $v^{[k]}$ the truncated divisor defined by

$$
v^{[k]}(z)=\min \{v(z), k\} \quad(z \in \mathbf{C})
$$

and put $N^{[k]}(r, v)=N\left(r, v^{[k]}\right)$. We will omit the character ${ }^{[k]}$ if $k=+\infty$.

Let $f$ be a meromorphic function on $\mathbf{C}$. We denote by $v_{f}^{0}\left(\right.$ resp. $\left.v_{f}^{\infty}\right)$ the zero divisor (resp. pole divisor) of $f$ and define the divisor generated by $f$ by $v_{f}=v_{f}^{0}-v_{f}^{\infty}$. The proximity function of $f$ is defined by

$$
m(r, f)=\frac{1}{2 \pi} \int_{0}^{2 \pi} \log ^{+}\left|\left(f\left(r e^{i \theta}\right)\right)\right| d \theta-\frac{1}{2 \pi} \int_{0}^{2 \pi} \log ^{+}\left|\left(f\left(e^{i \theta}\right)\right)\right| d \theta,
$$

2010 Mathematics Subject Classification. Primary 32H30, 32A22; Secondary 30D35.

Key words and phrases. Truncated multiplicity, holomorphic mapping, counting function, uniqueness.

Received October 9, 2012; revised March 26, 2013. 
where $\log ^{+} x=\max \{0, \log x\}$ for a positive number $x$. The Nevanlinna's characteristic function of $f$ is define by

$$
T(r, f)=m(r, f)+N\left(r, v_{f}^{\infty}\right) .
$$

In 1926, R. Nevanlinna proved the following theorem.

THEOREM A (Nevanlinna [3]). Let $f$ and $g$ be two distinct non-constant meromorphic functions and let $a_{1}, a_{2}, a_{3}, a_{4}$ be four distinct values in $\mathbf{C} \cup\{\infty\}$. Assume that

$$
v_{f-a_{i}}^{0}=v_{g-a_{i}}^{0} \quad \text { for } i=1, \ldots, 4 .
$$

Then $g$ is a Möbius transformation of $f$.

The above theorem is usually called the four values theorem of Nevanlinna. In 1983, Gundersen [1] improved the result of Nevanlinna by proving the following.

THEOREM B (Gundersen [1]). Let $f$ and $g$ be two distinct non-constant meromorphic functions and let $a_{1}, a_{2}, a_{3}, a_{4}$ be four distinct values in $\mathbf{C} \cup\{\infty\}$. Assume that

$$
\min \left\{v_{f-a_{i}}^{0}, 1\right\}=\min \left\{v_{g-a_{i}}^{0}, 1\right\} \quad \text { for } i=1,2 \quad \text { and } \quad v_{f-a_{j}}^{0}=v_{g-a_{j}}^{0} \text { for } j=3,4
$$

(outside a discrete set of counting function regardless of multiplicity is equal to $o(T(r, f)))$. Then $v_{f-a_{i}}^{0}=v_{g-a_{i}}^{0}$ for all $i \in\{1, \ldots, 4\}$.

Since that time, the above results of Nevanlinna and Gundersen have been extended and deepen by many authors for the case of meromorphic functions. However, so far as we know, there is no extension of such results to the case of holomorphic mappings into $\mathbf{P}^{n}(\mathbf{C})$. Our purpose in this paper is to generalize and improve these results to that case. To state our results, we give the following.

Firstly, we note that the condition: $\min \left\{k, v_{f-a_{i}}^{0}\right\}=\min \left\{k, v_{g-a_{i}}^{0}\right\}$ in Theorem A and Theorem B will implies that

$$
(*) f^{(l)}(z)=g^{(l)}(z) \quad \forall 0 \leq l<\min \left\{v_{f-a_{i}}^{0}(z), v_{g-a_{i}}^{0}(z)\right\}
$$

for all $z \in \operatorname{Supp} v_{f-a_{i}}^{0}=\operatorname{Supp} v_{g-a_{i}}^{0}$, where by $f^{(k)}$ we denote the $k$-th derivative of $f$ and $f^{(0)}=f$. Therefore, in a natural way, we generalize the notion "sharing a value" of meromorphic functions to the case of holomorphic mappings as follows.

Let $f$ and $g$ be two holomorphic mappings of $\mathbf{C}$ into $\mathbf{P}^{n}(\mathbf{C})$ with reduced representations

$$
f=\left(f_{0}: \cdots: f_{n}\right) \quad \text { and } \quad g=\left(g_{0}: \cdots: g_{n}\right)
$$


respectively. Let $H$ be a hyperplane in $\mathbf{P}^{n}(\mathbf{C})$ given by

$$
H=\left\{\left(\omega_{0}: \cdots: \omega_{n}\right): a_{0} \omega_{0}+\cdots+a_{n} \omega_{n}=0\right\},
$$

where $a_{i}(i=1, \ldots, n)$ are constants, not all zeros. We define functions $(f, H)=$ $\sum_{i=0}^{n} a_{i} f_{i}$ and $(g, H)=\sum_{i=0}^{n} a_{i} g_{i}$. Let $k$ be a positive integer or $+\infty$. We say that $f$ and $g$ share the hyperplane $H \mathrm{CM}_{k}^{*}$ if the following are satisfied

(i) $\min \left\{k, v_{(f, H)}^{0}(z)\right\}=\min \left\{k, v_{(g, H)}^{0}(z)\right\}$,

(ii) $\left(\frac{f_{i}}{f_{j}}\right)^{(l)}(z)=\left(\frac{g_{i}}{g_{j}}\right)^{(l)}(z) \quad \forall 0 \leq l<\min \left\{v_{(f, H)}^{0}(z), v_{(g, H)}^{0}(z)\right\}$,

for all $z$ outside a discrete set $S$ of counting function regardless of multiplicity equal to $o\left(T_{f}(r)+T_{g}(r)\right)$ ). If $k=1$ (resp. $\left.k=+\infty\right)$, we will write IM $^{*}$ (resp. $\mathrm{CM}^{*}$ ) for $\mathrm{CM}_{1}^{*}\left(\right.$ resp. $\left.\mathrm{CM}_{+\infty}^{*}\right)$. Omitting the condition on the derivative of $\frac{f_{i}}{f_{j}}$ and $\frac{g_{i}}{g_{j}}$, we say that $f$ and $g$
share $H \overline{\mathrm{CM}}_{k}^{*}$ if

$$
\min \left\{k, v_{(f, H)}^{0}(z)\right\}=\min \left\{k, v_{(g, H)}^{0}(z)\right\} \quad \text { and } \quad f(z)=g(z)
$$

for all $z \in f^{-1}(H) \cup g^{-1}(H) \backslash S$, where $S$ is a set of counting function regardless of multiplicity equal to $o\left(T_{f}(r)+T_{g}(r)\right)$ ). Similarly as above, if $k=1$ (resp. $k=+\infty)$, we will write $\overline{\mathrm{IM}}^{*}\left(\operatorname{resp} . \overline{\mathrm{CM}}^{*}\right)$ for $\overline{\mathrm{CM}}_{1}^{*}\left(\operatorname{resp} . \overline{\mathrm{CM}}_{+\infty}^{*}\right)$.

We now extend the results of Nevanlinna and Gundersen to the case of holomorphic mappings into $\mathbf{P}^{n}(\mathbf{C})$ as follows.

THEOREM 1.1. Let $f$ and $g$ be two linearly non-degenerate holomorphic mappings of $\mathbf{C}$ into $\mathbf{P}^{n}(\mathbf{C})$. Let $\left\{H_{i}\right\}_{i=1}^{2 n+2}$ be $(2 n+2)$ hyperplanes of $\mathbf{P}^{n}(\mathbf{C})$ in general position with $f^{-1}\left(H_{i}\right) \cap f^{-1}\left(H_{j}\right)=\emptyset(1 \leq i<j \leq 2 n+2)$. Assume that $f$ and $g$ share $(n+1)$ hyperplanes $\left\{H_{i}\right\}_{i=1}^{n+1} \mathrm{IM}^{*}$ and share $(n+1)$ hyperplanes $\left\{H_{i}\right\}_{i=n+2}^{2 n+2} \mathrm{CM}_{2}^{*}$. If $n \geq 2$ then $f=g$.

For the case of holomorphic mappings sharing $\overline{\mathrm{CM}}_{k}^{*}$ hyperplanes, we prove the following theorem.

THEOREM 1.2. Let $f$ and $g$ be two linearly non-degenerate holomorphic mappings of $\mathbf{C}$ into $\mathbf{P}^{n}(\mathbf{C})$. Let $\left\{H_{i}\right\}_{i=1}^{2 n+2}$ be $(2 n+2)$ hyperplanes of $\mathbf{P}^{n}(\mathbf{C})$ in general position with $f^{-1}\left(H_{i}\right) \cap f^{-1}\left(H_{j}\right)=\emptyset(1 \leq i<j \leq 2 n+2)$. Assume that $f$ and $g$ share $(n+1)$ hyperplanes $\left\{H_{i}\right\}_{i=1}^{n+1} \overline{\mathrm{IM}}^{*}$ and share $(n+1)$ hyperplanes $\left\{H_{i}\right\}_{i=n+2}^{2 n+2} \overline{\mathrm{CM}}_{n+1}^{*}$. If $n \geq 2$ then $f=g$.

\section{Basic notions in Nevanlinna theory}

Let $f: \mathbf{C} \rightarrow \mathbf{P}^{n}(\mathbf{C})$ be a holomorphic mapping. For arbitrarily fixed homogeneous coordinates $\left(w_{0}: \cdots: w_{n}\right)$ on $\mathbf{P}^{n}(\mathbf{C})$, we take a reduced representation 
$f=\left(f_{0}: \cdots: f_{n}\right)$, which means that $f_{0}, \ldots, f_{n}$ are holomorphic functions on $\mathbf{C}$ have no common zero. Set $\|f\|=\left(\left|f_{0}\right|^{2}+\cdots+\left|f_{n}\right|^{2}\right)^{1 / 2}$.

The characteristic function of $f$ is defined by

$$
T_{f}(r)=\frac{1}{2 \pi} \int_{0}^{2 \pi} \log \left\|f\left(r e^{i \theta}\right)\right\| d \theta-\frac{1}{2 \pi} \int_{0}^{2 \pi} \log \left\|f\left(e^{i \theta}\right)\right\| d \theta .
$$

Let $\varphi$ be a nonzero meromorphic function on $\mathbf{C}$, which is occasionally regarded as a meromorphic map into $\mathbf{P}^{1}(\mathbf{C})$. Then, there is a fact that

$$
T_{\varphi}(r)=T(r, \varphi)+O(1) .
$$

The meromorphic function $\varphi$ is said to be small with respect to $f$ iff

$$
\| T(r, \varphi)=o\left(T_{f}(r)\right) .
$$

Here, as usual, by the notation " $\| P$ " we mean the assertion $P$ holds for all $r \in[0, \infty)$ excluding a Borel subset $E$ of the interval $[0, \infty)$ with $\int_{E} d r<\infty$. We denote by $\mathscr{R}_{f}$ the field of all small (with respect to $f$ ) functions on $\mathbf{C}$.

The following play essential roles in Nevanlinna theory.

Theorem 2.1 (Second main theorem, see [4]). Let $f: \mathbf{C} \rightarrow \mathbf{P}^{n}(\mathbf{C})$ be a linearly non-degenerate holomorphic mapping and $H_{1}, \ldots, H_{q}$ be $q$ hyperplanes in general position in $\mathbf{P}^{n}(\mathbf{C})$. Then

$$
\|(q-n-1) T_{f}(r) \leq \sum_{i=1}^{q} N^{[n]}\left(r, v_{\left(f, H_{i}\right)}^{0}\right)+o\left(T_{f}(r)\right) .
$$

Lemma 2.2 (Lemma on logarithmic derivative, see [4]). Let $f$ be a nonzero meromorphic function on $\mathbf{C}^{m}$. Then

$$
\| m\left(r, \frac{f^{(k)}}{f}\right)=O\left(\log ^{+} T(r, f)\right) \quad\left(k \in \mathbf{Z}_{+}\right) .
$$

The next lemma is due to P. Li and C. C. Yang.

Lemma 2.3 (Lemma 7, [2]). Let $f_{1}$ and $f_{2}$ be two non-constant meromorphic functions satisfying

$$
\| N^{[1]}\left(r, v_{f_{i}}^{0}\right)+N^{[1]}\left(r, v_{f_{i}}^{\infty}\right)=o\left(T\left(r, f_{1}\right)+T\left(r, f_{2}\right)\right) \quad(i=1,2) .
$$

If $\left(f_{1}^{s} f_{2}^{t}-1\right)$ is not identically zero for all integers $s$ and $t(|s|+|t|>0)$, then for any positive number $\varepsilon$, we have

$$
N_{0}\left(r, 1 ; f_{1}, f_{2}\right) \leq \varepsilon\left(T\left(r, f_{1}\right)+T\left(r, f_{2}\right)\right)
$$

where $N_{0}\left(r, 1 ; f_{1}, f_{2}\right)$ denotes the reduced counting function of $f_{1}$ and $f_{2}$ related to the common 1-points. 


\section{Some Lemmas}

In order to prove Main Theorems, we need some following lemmas.

LEMMA 3.1. Let $f$ and $g$ be two linearly nondegenerate holomorphic mappings of $\mathbf{C}$ into $\mathbf{P}^{n}(\mathbf{C})$ and let $\left\{H_{i}\right\}_{i=1}^{q}$ be q hyperplanes of $\mathbf{P}^{n}(\mathbf{C})$ in general position. Assume that $f$ and $g$ share $H_{i} \overline{\mathrm{IM}}^{*}$ for all $1 \leq i \leq q$. If $q \geq n+2$ then

$$
\| T_{g}(r)=O\left(T_{f}(r)\right) \quad \text { and } \quad \| T_{f}(r)=O\left(T_{g}(r)\right) .
$$

Proof. By the Second Main Theorem, we have

$$
\begin{aligned}
\|(q-n-1) T_{g}(r) & \leq \sum_{i=1}^{q} N^{[n]}\left(r, v_{\left(g, H_{i}\right)}^{0}\right)+o\left(T_{g}(r)\right) \leq \sum_{i=1}^{q} n N^{[1]}\left(r, v_{\left(g, H_{i}\right)}^{0}\right)+o\left(T_{g}(r)\right) \\
& =\sum_{i=1}^{q} n N^{[1]}\left(r, v_{\left(f, H_{i}\right)}^{0}\right)+o\left(T_{g}(r)\right) \leq q n T_{f}(r)+o\left(T_{g}(r)\right) .
\end{aligned}
$$

Hence $\| T_{g}(r)=O\left(T_{f}(r)\right)$. Similarly, we get $\| T_{f}(r)=O\left(T_{g}(r)\right)$.

Lemma 3.2. Let $f_{1}$ and $f_{2}$ be two distinct holomorphic mappings of $\mathbf{C}$ into $\mathbf{P}^{n}(\mathbf{C})$ and let $\left\{H_{i}\right\}_{i=1}^{2 n+2}$ be hyperplanes of $\mathbf{P}^{n}(\mathbf{C})$ in general position with $f_{1}^{-1}\left(H_{i}\right) \cap f_{1}^{-1}\left(H_{j}\right)=\emptyset(1 \leq i<j \leq 2 n+2)$. Suppose that $f_{1}$ and $f_{2}$ share all $H_{i}(1 \leq i \leq 2 n+2) \overline{\mathrm{IM}}^{*}$. Then the following assertions hold:

i) $\| T_{f_{s}}(r)=\frac{1}{n+1} \sum_{i=1}^{2 n+2} N^{[n]}\left(r, v_{\left(f_{s}, H_{i}\right)}^{0}\right)+S(r)$, for $\quad s=1,2 \quad$ and $\quad S(r)=$ $o\left(T_{f_{1}}(r)+T_{f_{2}}(r)\right)$.

ii) $\| N\left(r, \min \left\{v_{\left(f_{1}, H_{i}\right)}^{0}, v_{\left(f_{2}, H_{i}\right)}^{0}\right\}\right)=\sum_{s=1}^{2} N^{[n]}\left(r, v_{\left(f_{s}, H_{i}\right)}^{0}\right)-n N^{[1]}\left(r, v_{\left(f_{t}, H_{i}\right)}^{0}\right)+S(r)$

for $\forall 1 \leq t \leq 2,1 \leq i \leq 2 n+2$.
iii) For two indices $i, j(1 \leq i<j \leq 2 n+2)$, if $\frac{\left(f_{1}, H_{i}\right)}{\left(f_{2}, H_{i}\right)} \neq \frac{\left(f_{1}, H_{j}\right)}{\left(f_{2}, H_{j}\right)}$ then

$\|\left(T_{f_{1}}(r)+T_{f_{2}}(r)\right)=\sum_{v=i, j} N\left(r, \min \left\{v_{\left(f_{1}, H_{v}\right)}^{0}, v_{\left(f_{2}, H_{v}\right)}^{0}\right\}\right)+\sum_{\substack{v=1 \\ v \neq i, j}}^{2 n+2} N^{[1]}\left(r, v_{\left(f_{s}, H_{v}\right)}^{0}\right)+S(r)$

and $\| T\left(r, \frac{\left(f_{1}, H_{i}\right)}{\left(f_{1}, H_{j}\right)} / \frac{\left(f_{2}, H_{i}\right)}{\left(f_{2}, H_{j}\right)}\right)=\sum_{\substack{v=1 \\ v \neq i, j}}^{2 n+2} N^{[1]}\left(r, v_{\left(f_{s}, H_{v}\right)}^{0}\right)+S(r), \quad s=1,2$,

iv) For two indices $i, j(1 \leq i<j \leq 2 n+2)$, if $\frac{\left(f_{1}, H_{i}\right)}{\left(f_{2}, H_{i}\right)}=\frac{\left(f_{1}, H_{j}\right)}{\left(f_{2}, H_{j}\right)}$ then

$$
\| N^{[1]}\left(r, v_{\left(f_{s}, H_{i}\right)}^{0}\right)=N^{[1]}\left(r, v_{\left(f_{s}, H_{j}\right)}^{0}\right)+S(r)=S(r), \quad s=1,2,
$$


Proof. i)-ii) By changing indices if necessary, we may assume that

$$
\begin{gathered}
\underbrace{\frac{\left(f_{1}, H_{1}\right)}{\left(f_{2}, H_{1}\right)} \equiv \frac{\left(f_{1}, H_{2}\right)}{\left(f_{2}, H_{2}\right)} \equiv \cdots \equiv \frac{\left(f_{1}, H_{k_{1}}\right)}{\left(f_{2}, H_{k_{1}}\right)}}_{\text {group } 1} \not \equiv \underbrace{\frac{\left(f_{1}, H_{k_{1}+1}\right)}{\left(f_{2}, H_{k_{1}+1}\right)} \equiv \cdots \equiv \frac{\left(f_{1}, H_{k_{2}}\right)}{\left(f_{2}, H_{k_{2}}\right)}}_{\text {group 2 }} \\
\quad \neq \underbrace{\frac{\left(f_{1}, H_{k_{2}+1}\right)}{\left(f_{2}, H_{k_{2}+1}\right)} \equiv \cdots \equiv \frac{\left(f_{1}, H_{k_{3}}\right)}{\left(f_{2}, H_{k_{3}}\right)}}_{\text {group 3 }} \not \equiv \cdots \neq \underbrace{\frac{\left(f_{1}, H_{k_{m-1}+1}\right)}{\left(f_{2}, H_{k_{m-1}+1}\right)} \equiv \cdots \equiv \frac{\left(f_{1}, H_{k_{m}}\right)}{\left(f_{2}, H_{k_{m}}\right)}}_{\text {group } m},
\end{gathered}
$$

where $k_{m}=2 n+2$.

For each $1 \leq i \leq 2 n+2$, we set

$$
\sigma(i)= \begin{cases}i+n & \text { if } i+n \leq 2 n+2 \\ i-n-2 & \text { if } i+n>2 n+2\end{cases}
$$

and

$$
P_{i}=\left(f_{1}, H_{i}\right)\left(f_{2}, H_{\sigma(i)}\right)-\left(f_{2}, H_{i}\right)\left(f_{1}, H_{\sigma(i)}\right) .
$$

Since $f_{1}$ and $f_{2}$ are distinct maps, the number of elements of each group is at most $n$. Hence $\frac{\left(f_{1}, H_{i}\right)}{\left(f_{2}, H_{i}\right)}$ and $\frac{\left(f_{1}, H_{\sigma(i)}\right)}{\left(f_{2}, H_{\sigma(i)}\right)}$ belong to distinct groups. This means that $P_{i} \not \equiv 0(1 \leq i \leq 2 n+2)$.

For a fixed index $i(1 \leq i \leq 2 n+2)$, we easily see that:

- If $z \in f_{1}^{-1}\left(H_{i}\right) \cap f_{2}^{-1}\left(H_{i}\right)$ then $z$ is a zero point of $P_{i}$ with multiplicity at least $\min \left\{v_{\left(f_{1}, H_{i}\right)}^{0}(z), v_{\left(f_{2}, H_{i}\right)}^{0}(z)\right\}$. Similarly, if $z \in f_{1}^{-1}\left(H_{\sigma(i)}\right) \cap f_{2}^{-1}\left(H_{\sigma(i)}\right)$ then $z$ is a zero point of $P_{i}$ with multiplicity at least $\min \left\{v_{\left(f, H_{\sigma(i)}\right)}^{0}(z), v_{\left(g, H_{\sigma(i)}\right)}^{0}(z)\right\}$.

- If $z \in f_{1}^{-1}\left(H_{v}\right) \cap f_{2}^{-1}\left(H_{v}\right)$ with $v \notin\{i, \sigma(i)\}$ then $z$ is a zero point of $P_{i}$ (because $f_{1}(z)=f_{2}(z)$ ).

Thus, we have

$$
\begin{aligned}
v_{P_{i}}^{0}(z) \geq & \min \left\{v_{\left(f_{1}, H_{i}\right)}^{0}(z), v_{\left(f_{2}, H_{i}\right)}^{0}(z)\right\}+\min \left\{v_{\left(f_{1}, H_{\sigma(i)}\right)}^{0}(z), v_{\left(f_{2}, H_{\sigma(i)}\right)}^{0}(z)\right\} \\
& +\sum_{\substack{v=1 \\
v \neq i, \sigma(i)}}^{2 n+2} \min \left\{1, v_{\left(f_{s}, H_{v}\right)}^{0}(z)\right\}, \quad s=1,2,
\end{aligned}
$$

for all $z$ outside a discrete set of reduced counting function equal to $S(r)$.

Since $(\min \{a, b\} \geq \min \{a, n\}+\min \{b, n\}-n)$ for all positive integers $a$ and $b$, we have

$$
\begin{aligned}
v_{i}(z):= & \min \left\{v_{\left(f_{1}, H_{i}\right)}^{0}(z), v_{\left(f_{2}, H_{i}\right)}^{0}(z)\right\}-\min \left\{v_{\left(f_{1}, H_{i}\right)}^{0}(z), n\right\} \\
& -\min \left\{v_{\left(f_{2}, H_{i}\right)}^{0}(z), n\right\}-n \min \left\{v_{\left(f_{s}, H_{i}\right)}(z), 1\right\} \geq 0,
\end{aligned}
$$


for $s=1,2$, and for all $z$ outside a discrete set of reduced counting function equal to $S(r)$. Therefore, the inequality (3.3) implies that

$$
\begin{aligned}
v_{P_{i}}(z) \geq & \sum_{v=i, \sigma(i)}\left(\min \left\{v_{\left(f_{1}, H_{v}\right)}(z), n\right\}+\min \left\{v_{\left(f_{2}, H_{v}\right)}(z), n\right\}\right. \\
& \left.-n \min \left\{v_{\left(f_{s}, H_{v}\right)}(z), 1\right\}\right)+\sum_{\substack{v=1 \\
v \neq i, \sigma(i)}}^{2 n+2} \min \left\{v_{\left(f_{s}, H_{v}\right)}^{0}, 1\right\}(z),
\end{aligned}
$$

for $s=1,2$, and for all $z$ outside a discrete set of reduced counting function equal to $S(r)$.

Integrating both sides of the above inequality, we get

$$
\begin{aligned}
\| N_{P_{i}}(r) \geq & \sum_{v=i, \sigma(i)}\left(N_{\left(f_{1}, H_{v}\right)}^{[n]}(r)+N_{\left(f_{2}, H_{v}\right)}^{[n]}(r)-n N_{\left(f_{s}, H_{v}\right)}^{[1]}(r)\right) \\
& +\sum_{\substack{v=1 \\
v \neq i, \sigma(i)}}^{2 n+2} N_{\left(f_{s}, H_{v}\right)}^{[1]}(r)+S(r), \quad(s=1,2) .
\end{aligned}
$$

Summing-up both sides of this inequality over all $i=1, \ldots, n+1$, we have

$$
\| \sum_{i=1}^{n+1} N_{P_{i}}(r) \geq \sum_{s=1}^{2} \sum_{i=1}^{2 n+2} N_{\left(f_{s}, H_{v}\right)}^{[1]}(r)+S(r) .
$$

On the other hand, by Jensen's formula and the definition of the characteristic function, we have

$$
\begin{aligned}
N_{P_{i}}(r)= & \int_{0}^{2 \pi} \log \left|P_{i}\left(r e^{i \theta}\right)\right| d \theta+O(1) \\
\leq & \int_{0}^{2 \pi} \log \left(\left|\left(f_{1}, H_{i}\right)\left(r e^{i \theta}\right)\right|^{2}+\left|\left(f_{1}, H_{\sigma(i)}\right)\left(r e^{i \theta}\right)\right|^{2}\right)^{1 / 2} d \theta \\
& +\int_{0}^{2 \pi} \log \left(\left|\left(f_{2}, H_{i}\right)\left(r e^{i \theta}\right)\right|^{2}+\left|\left(f_{2}, H_{\sigma(i)}\right)\left(r e^{i \theta}\right)\right|^{2}\right)^{1 / 2} d \theta+O(1) \\
\leq & \int_{0}^{2 \pi} \log \left(\left\|f_{1}\left(r e^{i \theta}\right)\right\|\left(\left\|H_{i}\right\|^{2}+\left\|H_{\sigma(i)}\right\|^{2}\right)^{1 / 2}\right) d \theta \\
& +\int_{0}^{2 \pi} \log \left(\left\|f_{2}\left(r e^{i \theta}\right)\right\|\left(\left\|H_{i}\right\|^{2}+\left\|H_{\sigma(i)}\right\|^{2}\right)^{1 / 2}\right) d \theta+O(1) \\
= & \int_{0}^{2 \pi} \log \left\|f_{1}\left(r e^{i \theta}\right)\right\| d \theta+\int_{0}^{2 \pi} \log \left\|f_{2}\left(r e^{i \theta}\right)\right\| d \theta+O(1) \\
= & T_{f_{1}}(r)+T_{f_{2}}(r)+O(1) .
\end{aligned}
$$


This implies that

$$
(n+1)\left(T_{f_{1}}(r)+T_{f_{2}}(r)\right) \geq \sum_{s=1}^{2} \sum_{i=1}^{2 n+2} N_{\left(f_{s}, H_{i}\right)}^{[n]}(r)+S(r) .
$$

By the second main theorem, we also have

$$
\|(n+1) T_{f_{s}}(r) \leq \sum_{i=1}^{2 n+2} N_{\left(f_{s}, H_{i}\right)}^{[n]}(r)+S(r), \quad(s=1,2) .
$$

This implies that

$$
\|(n+1)\left(T_{f_{1}}(r)+T_{f_{2}}(r)\right) \leq \sum_{s=1}^{2} \sum_{i=1}^{2 n+2} N_{\left(f_{s}, H_{i}\right)}^{[n]}(r)+S(r) .
$$

This inequality yields that the inequality (3.7) becomes equality. Hence, it follows that all inequalities (3.3)-(3.5) become equalities for all $z$ outside a discrete set of reduced counting function equal to $S(r)$ and inequalities (3.6)-(3.9) become equality.

Therefore, (3.8) proves the assertion i) of the lemma. Integrating both sides of (3.4), we get the assertion ii) of the lemma.

iii) By re-changing index if necessary, without loss of generality we may assume that $j=\sigma(i)$. Since the assertion ii) holds and the inequality (3.6) becomes equality, we immediately get the first equality of the assertion iii). We now prove the second equality. We have

$$
\begin{aligned}
T\left(r, \frac{\left(f_{1}, H_{i}\right)}{\left(f_{1}, H_{j}\right)} / \frac{\left(f_{2}, H_{i}\right)}{\left(f_{2}, H_{j}\right)}\right)= & N\left(r, v_{\left(\left(f_{1}, H_{i}\right) /\left(f_{1}, H_{j}\right)\right) /\left(\left(f_{2}, H_{i}\right) /\left(f_{2}, H_{j}\right)\right)}^{\infty}\right) \\
& +m\left(r, \frac{\left(f_{1}, H_{i}\right)}{\left(f_{1}, H_{j}\right)} / \frac{\left(f_{2}, H_{i}\right)}{\left(f_{2}, H_{j}\right)}\right) \\
\leq & N\left(r, v_{\left(f_{1}, H_{i}\right) /\left(f_{2}, H_{i}\right)}^{\infty}\right)+N\left(r, v_{\left(f_{2}, H_{j}\right) /\left(f_{1}, H_{j}\right)}^{\infty}\right) \\
& +m\left(r, \frac{\left(f_{1}, H_{i}\right)}{\left(f_{1}, H_{j}\right)}\right)+m\left(r, \frac{\left(f_{2}, H_{j}\right)}{\left(f_{2}, H_{i}\right)}\right) \\
\leq & N\left(r, v_{\left(f_{1}, H_{i}\right) /\left(f_{2}, H_{i}\right)}^{\infty}\right)+N\left(r, v_{\left(f_{2}, H_{j}\right) /\left(f_{1}, H_{j}\right)}^{\infty}\right) \\
& +T\left(r, \frac{\left(f_{1}, H_{i}\right)}{\left(f_{1}, H_{j}\right)}\right)-N\left(r, v_{\left(f_{1}, H_{j}\right) /\left(f_{1}, H_{i}\right)}^{0}\right) \\
& +T\left(r, \frac{\left(f_{2}, H_{j}\right)}{\left(f_{2}, H_{i}\right)}\right)-N\left(r, v_{\left(f_{2}, H_{i}\right) /\left(f_{2}, H_{j}\right)}^{0}\right) \\
\leq & T_{f_{1}}(r)+T_{f_{2}(r)}(r) N\left(r, v_{\left(f_{1}, H_{i}\right) /\left(f_{2}, H_{i}\right)}^{\infty}\right) \\
& +N\left(r, v_{\left(f_{2}, H_{j}\right) /\left(f_{1}, H_{j}\right)}^{\infty}\right)-N\left(r, v_{\left(f_{1}, H_{j}\right)}^{0}\right)-N\left(r, v_{\left(f_{2}, H_{i}\right)}^{0}\right) .
\end{aligned}
$$


We see that $\min \left\{0, v_{\left(f_{2}, H_{i}\right)}^{0}(z)-v_{\left(f_{1}, H_{i}\right)}^{0}(z)\right\}-v_{\left(f_{2}, H_{i}\right)}^{0}(z)=-\min \left\{v_{\left(f_{1}, H_{i}\right)}^{0}(z)\right.$, $\left.v_{\left(f_{2}, H_{i}\right)}^{0}(z)\right\}$. Then it follows that

$$
N\left(r, v_{\left(f_{1}, H_{i}\right) /\left(f_{2}, H_{i}\right)}^{\infty}\right)-N\left(r, v_{\left(f_{2}, H_{i}\right)}^{0}\right)=-N\left(r, \min \left\{v_{\left(f_{1}, H_{i}\right)}^{0}, v_{\left(f_{2}, H_{i}\right)}^{0}\right\}\right) .
$$

Similarly, we have

$$
N\left(r, v_{\left(f_{2}, H_{j}\right) /\left(f_{1}, H_{j}\right)}^{\infty}\right)-N\left(r, v_{\left(f_{1}, H_{j}\right)}^{0}\right)=-N\left(r, \min \left\{v_{\left(f_{1}, H_{j}\right)}^{0}, v_{\left(f_{2}, H_{j}\right)}^{0}\right\}\right) .
$$

Combining these equalities and the above inequality, we obtain that

$$
\begin{aligned}
T\left(r, \frac{\left(f_{1}, H_{i}\right)}{\left(f_{1}, H_{j}\right)} / \frac{\left(f_{2}, H_{i}\right)}{\left(f_{2}, H_{j}\right)}\right) \leq & T_{f_{1}}(r)+T_{f_{2}}(r)-N\left(r, \min \left\{v_{\left(f_{1}, H_{i}\right)}^{0}, v_{\left(f_{2}, H_{i}\right)}^{0}\right\}\right) \\
& -N\left(r, \min \left\{v_{\left(f_{1}, H_{j}\right)}^{0}, v_{\left(f_{2}, H_{j}\right)}^{0}\right\}\right) .
\end{aligned}
$$

On the other hand, we see that

$$
\begin{aligned}
T\left(r, \frac{\left(f_{1}, H_{i}\right)}{\left(f_{1}, H_{j}\right)} / \frac{\left(f_{2}, H_{i}\right)}{\left(f_{2}, H_{j}\right)}\right) & \geq N\left(r, \frac{\left(f_{1}, H_{i}\right)}{\left(f_{1}, H_{j}\right)} / \frac{\left(f_{2}, H_{i}\right)}{\left(f_{2}, H_{j}\right)}-1\right) \\
& \geq \sum_{\substack{v=1 \\
v \neq i, j}}^{2 n+2} N^{[1]}\left(r, v_{\left(f_{s}, H_{v}\right)}^{0}\right), \quad s=1,2 .
\end{aligned}
$$

Combining this inequality, the inequality (3.10) and the first equality of the assertion, we obtain that

$$
\begin{aligned}
\| T\left(r, \frac{\left(f_{1}, H_{i}\right)}{\left(f_{1}, H_{j}\right)} / \frac{\left(f_{2}, H_{i}\right)}{\left(f_{2}, H_{j}\right)}\right) & =T_{f_{1}}(r)+T_{f_{2}}(r)-N\left(r, \min \left\{v_{\left(f_{1}, H_{i}\right)}^{0}, v_{\left(f_{2}, H_{i}\right)}^{0}\right\}\right)+S(r) \\
& =\sum_{\substack{v=1 \\
v \neq i, j}}^{2 n+2} N^{[1]}\left(r, v_{\left(f_{s}, H_{v}\right)}^{0}\right)+S(r), \quad s=1,2 .
\end{aligned}
$$

We get the last equality of the assertion iii).

iv) Suppose that $\frac{\left(f_{1}, H_{i}\right)}{\left(f_{1}, H_{j}\right)}=\frac{\left(f_{2}, H_{i}\right)}{\left(f_{2}, H_{j}\right)}$. Then $j \neq \sigma(i)$ and $i \neq \sigma(j)$. We also see that $v_{\left(f_{1}, H_{i}\right)}^{0}=v_{\left(f_{2}, H_{i}\right)}^{0}$ and $v_{\left(f_{1}, H_{\sigma(i)}\right)}^{0}=v_{\left(f_{2}, H_{\sigma(i)}\right)}^{0}$. We rewrite $P_{i}$ as follows

$$
P_{i}=\frac{\left(f_{1}, H_{i}\right)}{\left(f_{1}, H_{j}\right)}\left(\left(f_{1}, H_{j}\right)\left(f_{2}, H_{\sigma(i)}\right)-\left(f_{2}, H_{j}\right)\left(f_{1}, H_{\sigma(i)}\right)\right) .
$$

Therefore, if $z$ is a zero point of $\left(f_{1}, H_{i}\right)$ then $z$ is a zero point of $P_{i}$ with multiplicity at least $\left(v_{\left(f_{1}, H_{i}\right)}^{0}(z)+1\right)$. Hence, similarly as in the proof of assertions i) and ii) with this notice, we have 


$$
\begin{aligned}
\| N_{P_{i}}(r) \geq & \sum_{v=i, \sigma(i)}\left(N_{\left(f_{1}, H_{v}\right)}^{[n]}(r)+N_{\left(f_{2}, H_{v}\right)}^{[n]}(r)-n N_{\left(f_{s}, H_{v}\right)}^{[1]}(r)\right)+N_{\left(f_{s}, H_{i}\right)}^{[1]}(r) \\
& +\sum_{\substack{v=1 \\
v \neq i, \sigma(i)}}^{2 n+2} N_{\left(f_{s}, H_{v}\right)}^{[1]}(r)+S(r), \quad(s=1,2) .
\end{aligned}
$$

Since (3.6) becomes equality, the above inequality implies that

$$
\| N_{\left(f_{s}, H_{i}\right)}^{[1]}(r)=S(r), \quad s=1,2 .
$$

Similarly, we have $\| N_{\left(f_{s}, H_{j}\right)}^{[1]}(r)=S(r), s=1,2$. The assertion iii) is proved.

Lemma 3.11. Let $f_{1}$ and $f_{2}$ be two holomorphic mappings of $\mathbf{C}$ into $\mathbf{P}^{n}(\mathbf{C})$ and let $\left\{H_{i}\right\}_{i=1}^{2 n+2}$ be hyperplanes of $\mathbf{P}^{n}(\mathbf{C})$ in general position with $f_{1}^{-1}\left(H_{i}\right) \cap f_{1}^{-1}\left(H_{j}\right)=\emptyset(1 \leq i<j \leq 2 n+2)$. Assume that $f_{1}$ and $f_{2}$ share all $H_{i}(1 \leq i \leq n+1) \overline{\mathrm{IM}}^{*}$ and share $H_{i}(n+2 \leq i \leq 2 n+2) \overline{\mathrm{CM}}^{*}$. If $n \geq 2$ then $f_{1}=f_{2}$.

Proof. Suppose that $f_{1} \neq f_{2}$. By Lemma 3.1 we have $\| T_{f_{1}}(r)=$ $T_{f_{2}}(r)+S(r)$ and $\| T_{f_{2}}(r)=T_{f_{1}}(r)+S(r)$, where $S(r)=o\left(T_{f_{1}}(r)+T_{f_{2}}(r)\right)$. Set $h_{i}=\frac{\left(f_{1}, H_{i}\right)}{\left(f_{2}, H_{i}\right)}(i=1, \ldots, 2 n+2)$.

We distinguish the following two cases.

CASE 1. Assume that for every positive number $\varepsilon>0$ there exists a subset $E_{\varepsilon} \subset \mathbf{R}^{+}$with $\int_{E_{\varepsilon}} d t=+\infty$ such that

$$
\| \sum_{i=1}^{n+1} N^{[1]}\left(r, v_{f_{s}, H_{i}}^{0}\right) \leq \varepsilon T(r)+S(r), \quad s=1,2, \forall r \in E_{\varepsilon},
$$

where $T(r)=T_{f_{1}}(r)+T_{f_{2}}(r)$.

For each $j \in\{n+1, \ldots, 2 n+2\}$, by the second main theorem we have

$$
\begin{aligned}
\| T_{f_{s}}(r) & \leq N^{[n]}\left(r, v_{\left(f_{s}, H_{j}\right)}^{0}\right)+\sum_{i=1}^{n+1} N^{[n]}\left(r, v_{f_{s}, H_{i}}^{0}\right)+S(r) \\
& \leq N^{[n]}\left(r, v_{\left(f_{s}, H_{j}\right)}^{0}\right)+n \varepsilon T(r)+S(r), \quad s=1,2, \forall r \in E_{\varepsilon} .
\end{aligned}
$$

Since we suppose that $f_{1} \neq f_{2}$, there exist two indices $j_{1}, j_{2} \in\{n+1, \ldots, 2 n+2\}$ such that $P=\left(f_{1}, H_{j_{1}}\right)\left(f_{2}, H_{j_{2}}\right)-\left(f_{2}, H_{j_{1}}\right)\left(f_{1}, H_{j_{2}}\right) \neq 0$. Similarly as in the proof of Lemma 3.2, we have the following 
$\| T_{f_{1}}(r)+T_{f_{2}}(r) \geq N\left(r, v_{P}^{0}\right)$

$$
\begin{aligned}
& \geq \sum_{v=j_{1}, j_{2}} N\left(r, \min \left\{v_{\left(f_{1}, H_{v}\right)}^{0}, v_{\left(f_{2}, H_{v}\right)}^{0}\right\}\right)+\sum_{\substack{v=n+2 \\
v \neq j_{1}, j_{2}}}^{2 n+2} N^{[1]}\left(r, v_{\left(f_{s}, H_{v}\right)}^{0}\right) \\
& =\sum_{v=j_{1}, j_{2}} N^{[n]}\left(r, v_{\left(f_{s}, H_{v}\right)}^{0}\right)+\sum_{\substack{v=n+2 \\
v \neq j_{1}, j_{2}}}^{2 n+2} N^{[1]}\left(r, v_{\left(f_{s}, H_{v}\right)}^{0}\right) \\
& \geq \sum_{v=j_{1}, j_{2}} N^{[n]}\left(r, v_{\left(f_{s}, H_{v}\right)}^{0}\right)+\frac{1}{n} \sum_{\substack{v=n+2 \\
v \neq j_{1}, j_{2}}}^{2 n+2} N^{[n]}\left(r, v_{\left(f_{s}, H_{v}\right)}^{0}\right) \\
& \geq\left(2+\frac{n-1}{n}\right)\left(T_{f_{s}}(r)-n \varepsilon T(r)\right)+S(r), \quad s=1,2, \forall r \in E_{\varepsilon} .
\end{aligned}
$$

Thus

$\| T_{f_{1}}(r)+T_{f_{2}}(r) \geq\left(1+\frac{n-1}{2 n}-\frac{3 n-1}{n} \cdot \varepsilon\right)\left(T_{f_{1}}(r)+T_{f_{2}}(r)\right)+S(r), \quad \forall \varepsilon>0, r \in E_{\varepsilon}$.

This implies that

$$
\| T_{f_{1}}(r)+T_{f_{2}}(r)=S(r), \quad\left(r \in E_{\varepsilon}\right)
$$

for all $\varepsilon<\frac{n-1}{2(3 n-1)}$. This is a contradiction.

CAse 2. Assume that there exists a positive number $\varepsilon_{0}>0$ such that

$$
\| \sum_{i=1}^{n+1} N^{[1]}\left(r, v_{\left(f_{s}, H_{i}\right)}^{0}\right) \geq \varepsilon_{0} T(r)+S(r), \quad s=1,2 .
$$

Set $h_{i}=\frac{\left(f_{1}, H_{i}\right)}{\left(f_{2}, H_{i}\right)}, i=1, \ldots, 2 n+2$.

Fix indices $i, j \in\{n+2, \ldots, 2 n+2\}$. By the supposition $f \neq g$, there exists an index $k \notin\{i, j\}$, such that $h_{i} \neq h_{k} \neq h_{j}$. Then from the first equality of the Lemma 3.2iii), we easily see that

$$
\begin{gathered}
\| N\left(r, \min \left\{v_{\left(f_{1}, H_{i}\right)}^{0}, v_{\left(f_{2}, H_{i}\right)}^{0}\right\}\right)+N\left(r, \min \left\{v_{\left(f_{1}, H_{k}\right)}^{0}, v_{\left(f_{2}, H_{k}\right)}^{0}\right\}\right) \\
=N\left(r, \min \left\{v_{\left(f_{1}, H_{j}\right)}^{0}, v_{\left(f_{2}, H_{j}\right)}^{0}\right\}\right)+N\left(r, \min \left\{v_{\left(f_{1}, H_{k}\right)}^{0}, v_{\left(f_{2}, H_{k}\right)}^{0}\right\}\right)+S(r) \\
\Rightarrow \| N\left(r, \min \left\{v_{\left(f_{1}, H_{i}\right)}^{0}, v_{\left(f_{2}, H_{i}\right)}^{0}\right\}\right)=N\left(r, \min \left\{v_{\left(f_{1}, H_{j}\right)}^{0}, v_{\left(f_{2}, H_{j}\right)}^{0}\right\}\right)+S(r) \\
\Rightarrow \| N\left(r, v_{\left(f_{s}, H_{i}\right)}^{0}\right)=N\left(r, v_{\left(f_{s}, H_{j}\right)}^{0}\right)+S(r), \quad s=1,2 .
\end{gathered}
$$


We see that the last inequality actually holds for all $i, j \in\{n+2, \ldots, 2 n+2\}$. Then there is a positive function $N(r)(r>1)$ such that

$$
\| N\left(r, v_{\left(f_{s}, H_{i}\right)}^{0}\right)=N(r)+S(r), \quad s=1,2 \text { and } i \in\{n+2, \ldots, 2 n+2\} .
$$

On the other hand, for a fixed index $i \in\{n+2, \ldots, 2 n+2\}$ as above, we repeat the same arguments as in the proof of Lemma 3.2i)-ii) and see that all inequalities (3.3)-(3.9) become equalities (maybe outside a discrete set of reduced counting function of $S(r)$ ). Then from (3.4), we have

$$
\begin{aligned}
\min \left\{v_{\left(f_{1}, H_{i}\right)}^{0}(z), v_{\left(f_{2}, H_{i}\right)}^{0}(z)\right\}= & \min \left\{v_{\left(f_{1}, H_{i}\right)}^{0}(z), n\right\}+\min \left\{v_{\left(f_{2}, H_{i}\right)}^{0}(z), n\right\} \\
& -n \min \left\{v_{\left(f_{s}, H_{i}\right)}(z), 1\right\} \\
\Leftrightarrow & \min \left\{v_{\left(f_{1}, H_{i}\right)}^{0}(z), v_{\left(f_{2}, H_{i}\right)}^{0}(z)\right\} \leq n \leq \max \left\{v_{\left(f_{1}, H_{i}\right)}^{0}(z), v_{\left(f_{2}, H_{i}\right)}^{0}(z)\right\} \\
\Leftrightarrow & v_{\left(f_{s}, H_{i}\right)}^{0}(z)=n, \quad s=1,2
\end{aligned}
$$

This implies that

$$
\begin{aligned}
\| n N^{[1]}\left(r, v_{\left(f_{s}, H_{i}\right)}^{0}\right)=N^{[n]}\left(r, v_{\left(f_{s}, H_{i}\right)}^{0}\right)+S(r) & =N\left(r, v_{\left(f_{s}, H_{i}\right)}^{0}\right)+S(r) \\
& =N(r)+S(r), \quad s=1,2 .
\end{aligned}
$$

for all $z \in f_{1}^{-1}\left(H_{i}\right) \cup f_{2}^{-1}\left(H_{i}\right) \backslash\{$ a discrete set of reduced counting function equal to $S(r)\}$.

Moreover, suppose that for every $\varepsilon>0$ there exists a subset $E_{\varepsilon} \subset \mathbf{R}^{+}$with $\int_{E_{\varepsilon}} d t=+\infty$ such that $\| N(r) \leq \varepsilon T(r)+S(r)$ for all $r \in E_{\varepsilon}$. Then we have

$$
\| \sum_{v=n+2}^{2 n+2} N^{[1]}\left(r, v_{\left(f_{s}, H_{v}\right)}^{0}\right)=(n+1) N(r)+S(r) \leq(n+1) \varepsilon T(r)+S(r)
$$

for $s=1,2$ and $r \in E_{\varepsilon}$. Hence, by repeating the same argument as in Case 1, we again get a contradiction. Therefore, there exists a positive number $\varepsilon_{1}>0$ such that

$$
\| N(r) \geq \varepsilon_{1} T(r)+S(r) .
$$

Suppose that there exist three indices $i_{1}, i_{2}, i_{3} \in\{n+2, \ldots, 2 n+2\}$ with $h_{i_{1}} \neq$ $\begin{array}{ll}h_{i_{2}} \neq h_{i_{3}} \neq h_{i_{1}} . & \text { By Lemma } 3.2 \mathrm{iii}) \text { we see that } \| T\left(r, \frac{h_{i_{v}}}{h_{i_{u}}}\right) \geq\left(\varepsilon_{0}+(n-1) \varepsilon_{1}\right) T(r), \\ 1 \leq u \neq v \leq 3 . & \text { We also have }\end{array}$

$$
\| N\left(r, v_{h_{i_{1}} / h_{i_{v}}}^{0}\right)+N\left(r, v_{h_{i_{1}} / h_{i_{v}}}^{\infty}\right)=S(r), \quad v=2,3,
$$

and $\| N_{0}\left(r ; 1, \frac{h_{i_{1}}}{h_{i_{2}}}, \frac{h_{i_{3}}}{h_{i_{1}}}\right) \geq \sum_{j=1}^{n+1} N^{[1]}\left(r, v_{\left(f_{1}, H_{j}\right)}^{0}\right)+S(r) \geq \varepsilon_{0} T(r)+S(r)$. 
Then Lemma 2.3 yields that there exist integers $p, q$, not all zeros, such that

$$
\left(\frac{h_{i_{1}}}{h_{i_{2}}}\right)^{p}=\left(\frac{h_{i_{1}}}{h_{i_{3}}}\right)^{q} \text {. }
$$

By Lemma 3.2iii) we have the following

$$
\begin{gathered}
T\left(r,\left(\frac{h_{i_{1}}}{h_{i_{2}}}\right)^{p}\right)=T\left(r,\left(\frac{h_{i_{1}}}{h_{i_{3}}}\right)^{q}\right) \\
\Leftrightarrow|p| T\left(r, \frac{h_{i_{1}}}{h_{i_{2}}}\right)=|q| T\left(r, \frac{h_{i_{1}}}{h_{i_{3}}}\right) \\
\Leftrightarrow|p| \sum_{j=1}^{n+1} N^{[1]}\left(r, v_{\left(f_{1}, H_{j}\right)}^{0}\right)+|p| \sum_{\substack{j=n+2 \\
j \neq i_{1}, i_{2}}}^{2 n+2} N^{[1]}\left(r, v_{\left(f_{1}, H_{i}\right)}^{0}\right) \\
=|q| \sum_{j=1}^{n+1} N^{[1]}\left(r, v_{\left(f_{1}, H_{j}\right)}^{0}\right)+|p| \sum_{\substack{j=n+2 \\
j \neq i_{1}, i_{3}}}^{2 n+2} N^{[1]}\left(r, v_{\left(f_{1}, H_{i}\right)}^{0}\right) \\
\Leftrightarrow(|p|-|q|)\left(\sum_{j=1}^{n+1} N^{[1]}\left(r, v_{\left(f_{1}, H_{j}\right)}^{0}\right)+\frac{n-1}{n} N(r)\right)=S(r) .
\end{gathered}
$$

This implies that $|p|=|q| \neq 0$. Suppossing that $p=-q$, we have

$$
\left(\frac{h_{i_{1}}}{h_{i_{2}}}\right)^{2 p}=\left(\frac{h_{i_{3}}}{h_{i_{2}}}\right)^{p} \text {. }
$$

Similarly to $(3.15)$, one gets

$$
(|2 p|-|p|)\left(\sum_{j=1}^{n+1} N^{[1]}\left(r, v_{\left(f_{1}, H_{j}\right)}^{0}\right)+\frac{n-1}{n} N(r)\right)=S(r) .
$$

Then $p=0$. This is a contradiction. Therefore, we must have $p=q \neq 0$. This implies that $h_{i_{2}}^{p}=h_{i_{3}}^{p}$. Hence $\frac{h_{i_{2}}}{h_{i_{3}}}=$ constant. This contradicts to the fact
that

$$
\begin{aligned}
\| T\left(r, \frac{h_{i_{2}}}{h_{i_{3}}}\right) & =\sum_{j=1}^{n+1} N^{[1]}\left(r, v_{\left(f_{1}, H_{j}\right)}^{0}\right)+|p| \sum_{\substack{j=n+2 \\
j \neq i_{2}, i_{3}}}^{2 n+2} N^{[1]}\left(r, v_{\left(f_{1}, H_{i}\right)}^{0}\right)+S(r) \\
& \geq \varepsilon_{0} T(r)+|p|(n-1) N(r)+S(r) .
\end{aligned}
$$

Then the supposition that $h_{i_{1}} \neq h_{i_{2}} \neq h_{i_{3}} \neq h_{i_{1}}$ is untrue. Therefore we mus have $h_{i_{1}}=h_{i_{2}}$ or $h_{i_{2}}=h_{i_{3}}$ or $h_{i_{3}}=h_{i_{1}}$. By this and by Lemma 3.2iv), we see that $\| N(r)=S(r)$. This is a contradiction. 
Hence the supposition $f_{1} \neq f_{2}$ is untrue. Therefore we have $f_{1}=f_{2}$. The lemma is proved.

\section{Proofs of Main Theorems}

Proof of Theorem 1.1. Suppose that $f \neq g$. By Lemma 3.2, we have $\| T_{f}(r)=O\left(T_{g}(r)\right)+S(r)$ and $\| T_{g}(r)=O\left(T_{f}(r)\right)+S(r)$, where $S(r)=o\left(T_{f}(r)+\right.$ $\left.T_{g}(r)\right)$.

Fix an index $i_{0} \in\{n+2, \ldots, 2 n+2\}$. Since $f \neq g$, there exist two indices $i, j \in\{1, \ldots, 2 n+2\} \backslash\left\{i_{0}\right\}$ such that $\frac{\left(f, H_{i}\right)}{\left(f, H_{j}\right)} \neq \frac{\left(g, H_{i}\right)}{\left(g, H_{j}\right)}$. Then by Lemma 3.2 (the last equality of the assertion iii)), we have

$$
\| T\left(r, \frac{\left(f, H_{i}\right)}{\left(f, H_{j}\right)} / \frac{\left(g, H_{i}\right)}{\left(g, H_{j}\right)}\right)=\sum_{\substack{v=1 \\ v \neq i, j}}^{2 n+2} N^{[1]}\left(r, v_{\left(u, H_{v}\right)}^{0}\right)+S(r), \quad u=f, g .
$$

On the other hand, since $f$ and $g$ share $H_{v}(v \neq i, j) \quad \mathrm{IM}^{*}$, every point $z \in f^{-1}\left(H_{v}\right) \cup g^{-1}\left(H_{v}\right)$ (outside a discrete set of reduced counting function equal to $S(r))$ is zero of $\left(\frac{\left(f, H_{i}\right)}{\left(f, H_{j}\right)} / \frac{\left(g, H_{i}\right)}{\left(g, H_{j}\right)}-1\right)$ with multiplicity at least $\min \left\{v_{\left(f, H_{v}\right)}^{0}(z), v_{\left(g, H_{v}\right)}^{0}(z)\right\}$. Therefore, we have

$$
\| T\left(r, \frac{\left(f, H_{i}\right)}{\left(f, H_{j}\right)} / \frac{\left(g, H_{i}\right)}{\left(g, H_{j}\right)}\right) \geq \sum_{\substack{v=1 \\ v \neq i, j}}^{2 n+2} N\left(r, \min \left\{v_{\left(f, H_{v}\right)}^{0}, v_{\left(g, H_{v}\right)}^{0}\right\}\right)+S(r) .
$$

Thus

$$
\| \sum_{\substack{v=1 \\ v \neq i, j}}^{2 n+2} N^{[1]}\left(r, v_{\left(u, H_{v}\right)}^{0}\right) \geq \sum_{\substack{v=1 \\ v \neq i, j}}^{2 n+2} N\left(r, \min \left\{v_{\left(f, H_{v}\right)}^{0}, v_{\left(g, H_{v}\right)}^{0}\right\}\right)+S(r), \quad s=1,2 .
$$

Because $\| N^{[1]}\left(r, v_{\left(u, H_{v}\right)}^{0}\right) \leq N\left(r, \min \left\{v_{\left(f, H_{v}\right)}^{0}, v_{\left(g, H_{v}\right)}^{0}\right\}\right)+S(r)$ for $u \in\{f, g\}$ and $v \in\{1, \ldots, 2 n+2\}$, then the above equality implies that $\| N^{[1]}\left(r, v_{\left(u, H_{v}\right)}^{0}\right)=N\left(r, \min \left\{v_{\left(f, H_{v}\right)}^{0}, v_{\left(g, H_{v}\right)}^{0}\right\}\right)+S(r)$ for $v \notin\{i, j\}$ and $u \in\{f, g\}$.

In particular, we have

(4.1) $\| N^{[1]}\left(r, v_{\left(f, H_{i_{0}}\right)}^{0}\right)=N^{[1]}\left(r, v_{\left(g, H_{i_{0}}\right)}^{0}\right)=N\left(r, \min \left\{v_{\left(f, H_{i_{0}}\right)}^{0}, v_{\left(g, H_{i_{0}}\right)}^{0}\right\}\right)+S(r)$.

These equality yield that

$$
\min \left\{v_{\left(f, H_{i_{0}}\right)}^{0}(z), v_{\left(g, H_{i_{0}}\right)}^{0}(z)\right\}=1
$$

for $\forall z \in f^{-1}\left(H_{i_{0}}\right) \cup g^{-1}\left(H_{i_{0}}\right) \backslash\{$ a discrete set of reduced counting function equal to $S(r)\}$. 
On the other hand, by Lemma 3.2ii) we have

$$
\begin{aligned}
N\left(r, \min \left\{v_{\left(f, H_{i_{0}}\right)}^{0}, v_{\left(g, H_{i_{0}}\right)}^{0}\right\}\right)= & N^{[n]}\left(r, v_{\left(f, H_{i_{0}}\right)}^{0}\right)+N^{[n]}\left(r, v_{\left(g, H_{i_{0}}\right)}^{0}\right) \\
& -n N^{[1]}\left(r, v_{\left(u, H_{i_{0}}\right)}^{0}\right), \quad \forall u=f, g .
\end{aligned}
$$

We also note that

$$
\begin{aligned}
\min \left\{v_{\left(f, H_{i_{0}}\right)}^{0}(z), v_{\left(g, H_{i_{0}}\right)}^{0}(z)\right\} \geq & \min \left\{n, v_{\left(f, H_{i_{0}}\right)}^{0}(z)\right\}+\min \left\{n, v_{\left(g, H_{i_{0}}\right)}^{0}(z)\right\} \\
& -n \min \left\{1, v_{\left(u, H_{i_{0}}\right)}^{0}(z)\right\}, \quad u=f, g,
\end{aligned}
$$

for all $z \in f^{-1}\left(H_{i_{0}}\right) \cap g^{-1}\left(H_{i_{0}}\right)$, and the inequality becomes equality if and only if

$$
\min \left\{v_{\left(f, H_{i_{0}}\right)}^{0}(z), v_{\left(g, H_{i_{0}}\right)}^{0}(z)\right\} \leq n \leq \max \left\{v_{\left(f, H_{i_{0}}\right)}^{0}(z), v_{\left(g, H_{i_{0}}\right)}^{0}(z)\right\} .
$$

Therefore, the equality (4.3) yields that

$$
\min \left\{v_{\left(f, H_{i_{0}}\right)}^{0}(z), v_{\left(g, H_{i_{0}}\right)}^{0}(z)\right\} \leq n \leq \max \left\{v_{\left(f, H_{i_{0}}\right)}^{0}(z), v_{\left(g, H_{i_{0}}\right)}^{0}(z)\right\}
$$

for $\forall z \in f^{-1}\left(H_{i_{0}}\right) \cup g^{-1}\left(H_{i_{0}}\right) \backslash$ a discrete set of reduced counting function equal to $S(r)\}$. From this and (4.2), we have

$$
\min \left\{v_{\left(f, H_{i_{0}}\right)}^{0}(z), v_{\left(g, H_{i_{0}}\right)}^{0}(z)\right\}=1 \text { and } \max \left\{v_{\left(f, H_{i_{0}}\right)}^{0}(z), v_{\left(g, H_{i_{0}}\right)}^{0}(z)\right\} \geq n
$$

for $\forall z \in f^{-1}\left(H_{i_{0}}\right) \cup g^{-1}\left(H_{i_{0}}\right) \backslash$ a discrete set of reduced counting function equal to $S(r)\}$

On the other hand we notice that: Since $f$ and $g$ share $H_{i_{0}} \mathrm{IM}_{2}^{*}$, if a point $z \in f^{-1}\left(H_{i_{0}}\right) \cup g^{-1}\left(H_{i_{0}}\right)$ (maybe outside a discrete set of reduced counting function equal to $S(r))$ is a zero of $\left(f_{1}, H_{i_{0}}\right)$ with multiplicity 1 then it will be zero of $\left(f_{2}, H_{i_{0}}\right)$ with multiplicity 1 and vise versa. This means that

$$
\max \left\{v_{\left(f, H_{i_{0}}\right)}^{0}(z), v_{\left(g, H_{i_{0}}\right)}^{0}(z)\right\}<n
$$

for $\forall z \in f^{-1}\left(H_{i_{0}}\right) \cup g^{-1}\left(H_{i_{0}}\right) \backslash$ a discrete set of reduced counting function equal to $S(r)\}$.

From this notice and (4.4), we easily see that

$$
\| N^{[1]}\left(r, v_{\left(u, H_{i_{0}}\right)}^{0}\right)=S(r), \quad u=f, g .
$$

Therefore, $f$ and $g$ share $H_{i_{0}} \overline{\mathrm{CM}}^{*}$. This holds for all $i_{0} \in\{n+2, \ldots, 2 n+2\}$. With the help of Lemma 3.11, we have $f=g$. This contradicts to the supposition.

Then we must have $f=g$. The theorem is proved.

Proof of Theorem 1.2. Suppose that $f \neq g$. As in the proof of Theorem 1.1, we have $\| T_{f}(r)=O\left(T_{g}(r)\right)+S(r)$ and $\| T_{g}(r)=O\left(T_{f}(r)\right)+S(r)$, where $S(r)=o\left(T_{f}(r)+T_{g}(r)\right)$. 
Fix an index $i_{0} \in\{n+2, \ldots, 2 n+2\}$. By Lemma 3.2ii) we have

$$
\begin{aligned}
N\left(r, \min \left\{v_{\left(f, H_{i_{0}}\right)}^{0}, v_{\left(g, H_{i_{0}}\right)}^{0}\right\}\right)= & N^{[n]}\left(r, v_{\left(f, H_{i_{0}}\right)}^{0}\right)+N^{[n]}\left(r, v_{\left(g, H_{i_{0}}\right)}^{0}\right) \\
& -n N^{[1]}\left(r, v_{\left(u, H_{i_{0}}\right)}^{0}\right), \quad u=f, g .
\end{aligned}
$$

Repeating the same argument as in the proof of Theorem 1, we see that

$$
\begin{aligned}
\min \left\{v_{\left(f, H_{i_{0}}\right)}^{0}(z), v_{\left(g, H_{i_{0}}\right)}^{0}(z)\right\} \geq & \min \left\{n, v_{\left(f, H_{i_{0}}\right)}^{0}(z)\right\}+\min \left\{n, v_{\left(g, H_{i_{0}}\right)}^{0}(z)\right\} \\
& -n \min \left\{1, v_{\left(u, H_{i_{0}}\right)}^{0}(z)\right\}, \quad u=f, g,
\end{aligned}
$$

for all $z \in f^{-1}\left(H_{i_{0}}\right) \cap g^{-1}\left(H_{i_{0}}\right)$, and this inequality becomes equality if and only if

$$
\min \left\{v_{\left(f, H_{i_{0}}\right)}^{0}(z), v_{\left(g, H_{i_{0}}\right)}^{0}(z)\right\} \leq n \leq \max \left\{v_{\left(f, H_{i_{0}}\right)}^{0}(z), v_{\left(g, H_{i_{0}}\right)}^{0}(z)\right\} .
$$

Then the equality (4.5) implies that

$$
\min \left\{v_{\left(f, H_{i_{0}}\right)}^{0}(z), v_{\left(g, H_{i_{0}}\right)}^{0}(z)\right\} \leq n \leq \max \left\{v_{\left(f, H_{i_{0}}\right)}^{0}(z), v_{\left(g, H_{i_{0}}\right)}^{0}(z)\right\}
$$

for $\forall z \in f^{-1}\left(H_{i_{0}}\right) \cup g^{-1}\left(H_{i_{0}}\right) \backslash$ a discrete set of reduced counting function equal to $S(r)\}$.

We also note that: Since $f$ and $g$ share $H_{i_{0}} \overline{\mathrm{IM}}_{n+1}^{*}$, if a point $z \in f^{-1}\left(H_{i_{0}}\right)$ $\cup g^{-1}\left(H_{i_{0}}\right)$ (maybe outside a discrete set of reduced counting function equal to $S(r))$ is a zero of $\left(f_{1}, H_{i_{0}}\right)$ with multiplicity $<n$ (resp. multiplicity $\left.>n\right)$ then it will be zero of $\left(f_{2}, H_{i_{0}}\right)$ with multiplicity $<n$ (resp. multiplicity $\left.>n\right)$ and vise versa.

From this notice and (4.6), we easily see that

$$
v_{\left(f, H_{i_{0}}\right)}^{0}(z)=v_{\left(g, H_{i_{0}}\right)}^{0}(z)=n
$$

for $\forall z \in f^{-1}\left(H_{i_{0}}\right) \cup g^{-1}\left(H_{i_{0}}\right) \backslash$ a discrete set of reduced counting function equal to $S(r)\}$.

Therefore, $f$ and $g$ share $H_{i_{0}} \overline{\mathrm{CM}}^{*}$. This holds for all $i_{0} \in\{n+2, \ldots, 2 n+2\}$. From Lemma 3.11, we have $f=g$. The theorem is proved.

Acknowledgements. This work was completed while the author was staying at Vietnam Institute for Advanced Study in Mathematics. He would like to thank the institute for their support. The research of the author was partially supported by a NAFOSTED grant of Vietnam.

\section{REFERENCES}

[ 1] G. Gundersen, Meromorphic functions that share four values, Trans. Amer. J. Math. 227 (1983), 545-567.

[2] P. LI AND C. C. YANG, On the characteristics of meromorphic functions that share three values CM, J. Math. Anal. Appl. 220 (1998), 135-145. 
[3] R. Nevanlinna, Einige Eideutigkeitssätze in der Theorie der meromorphen Funktionen, Acta. Math. 48 (1926), 367-391.

[4] J. Noguchi AND T. OChiaI, Introduction to geometric function theory in several complex variables, Trans. Math. Monogr. 80, Amer. Math. Soc., Providence, Rhode Island, 1990.

Duc Quang Si

Department of Mathematics

Hanoi National University of Education

136-Xuan Thuy, Cau Giay, HanoI

VIETNAM

E-mail: quangsd@hnue.edu.vn 